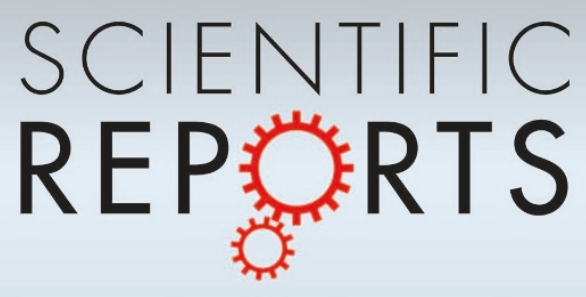

OPEN

SUBJECT AREAS:

NONLINEAR OPTICS

OPTOELECTRONIC DEVICES AND

COMPONENTS

TERAHERTZ OPTICS

Received

8 July 2014

Accepted

19 December 2014

Published

27 January 2015

Correspondence and requests for materials should be addressed to M.S. (most.shalaby@ gmail.com) or C.P.H. (christoph.hauri@psi.

\section{Spectrally intense terahertz source based on triangular Selenium}

\author{
Mostafa Shalaby ${ }^{1,2} \&$ Christoph P. Hauri ${ }^{1,3}$ \\ ${ }^{1}$ SwissFEL, Paul Scherrer Institute, 5232 Villigen PSI, Switzerland, ²Physics Department, ETH Zurich, 8093 Zurich, Switzerland, \\ ${ }^{3}$ Ecole Polytechnique Federale de Lausanne, 1015 Lausanne, Switzerland.
}

The intensity of a nonlinear terahertz $(\mathrm{THz})$ source is primarily given by its spectral density. In this letter, we introduce triangular Selinium (Se) as a novel $\mathrm{THz}$ emitter and show numerically its superior properties to the currently used crystals for intense $\mathrm{THz}$ generation. The excellent phase matching enables the applicability of elongated Se crystals which results in very high spectral flatness and broad THz bandwidth $(0.5-3.5 \mathrm{THz})$, high conversion efficiency and $\mathrm{THz}$ pulse energy. The spectral $\mathrm{THz}$ density produced by optical rectification in Selenium exceeds those from contemporary crystal-based THz sources.

$\mathrm{O}$ ngoing progress in $\mathrm{THz}(0.1-10 \mathrm{THz})$ source technology is to be credited for recent advances in nonlinear THz science ${ }^{1-3}$. Currently, electron accelerators ${ }^{4}$ and lasers are the main platforms for producing intense $\mathrm{THz}$ radiation but primarily laser-driven $\mathrm{THz}$ sources offer the versatility, stability and repetition rate desired in time-resolved investigations. Their performance, however, still lags behind the demands of many potential experiments in terms of spectral intensity, spectral tunability and arbitrary temporal pulse shaping ${ }^{5-6}$. The large diversity of applications requires versatile sources covering narrowband/broadband sources at variable central frequencies. While narrowband sources are a formidable tool for controlling collective motions in solids, for example, by selective excitation of resonant modes, pedestal-free single-cycle THz transients are beneficial for high-field applications. The latter requires, in principle, a smooth, modulation-free spectral shape. Presently, conventional $\mathrm{THz}$ sources cannot cover all of the manifold requirements in view of spectral intensity, spectral width and field strength which justifies the exploration of novel source schemes.

Different techniques have been employed in the past for $\mathrm{THz}$ generation driven by an intense femtosecond laser pulse. Broadband $\mathrm{THz}$ continuum was observed from a laser-induced plasma ${ }^{7}$, covering a large frequency band around $6 \mathrm{THz}$ but at low conversion efficiency $\left(10^{-4}\right)$ though. Optical rectification $(\mathrm{OR})$-based sources with Lithium Niobate $(\mathrm{LN})^{8}$ and organic crystals (e.g. DAST, DSTMS) ${ }^{9-11}$ show significantly higher conversion efficiencies (in the range of a percent) and deliver broadband radiation around 0.5 and $3 \mathrm{THz}$, respectively. The spectral density is quite strongly modulated due to phonon resonances, which hampers the formation of clean single-cycle $\mathrm{THz}$ pulses.

From a physical point of view OR is restricted primarily to thin nonlinear crystals and thus short interaction length, as temporal pump pulse broadening caused by the dispersion hampers efficient conversion. In this report, we present numerical investigations on a novel highly efficient $\mathrm{THz}$ source where high $\mathrm{THz}$ pulse energy is achieved by phase-matching of pump and THz radiation in triangular Se. This approach overcomes the shortcomings of limited pump-THz pulse interaction length mentioned above to a great extent. The reported crystal exhibits outstanding phase matching properties which allows to expand the longitudinal laser-crystal interaction length significantly while keeping the pump and $\mathrm{THz}$ beam temporally synchronized. Selenium offers a remarkably low absorption for $\mathrm{THz}$ and pump wavelengths $\left(8.1 \mathrm{~cm}^{-112}\right.$ at $\left.2 \mathrm{THz}\right)$ which is superior to other commonly used THz emitters (LN $39 \mathrm{~cm}^{-113}$; DAST $28 \mathrm{~cm}^{-114}$ ). Furthermore it shows outstanding large spectral intensities and offers the ability to generate flat-top, modulation-free THz spectra. Phase-matching is achieved for a large pump wavelength range in the short-wavelength infrared spectral region $(\lambda=1.1-2.7 \mu \mathrm{m})$. These attributes make the Se-based THz source attractive for future applications covering shortages of modern laser-based $\mathrm{THz}$ sources in the $0.1-3 \mathrm{THz}$ frequency range.

\section{Results}

Generation efficiency. The conversion efficiency of optical photons into the terahertz frequency range through OR depends primarily on the fundamental laser characteristics and properties of the nonlinear crystal at both optical and $\mathrm{THz}$ frequencies. In its simple form (that is in the plane wave, non-depleted pump approximation, and 
neglecting both pump absorption and higher order nonlinear effects), far from phonon resonances, the generated $\mathrm{THz}$ field $E(\omega$, $z$ ) through OR in a noncentrosymmetric crystal can be described ${ }^{15}$ by

$$
E(\omega, \mathrm{z})=\left(2 \mu_{0}\right) \mathrm{t}_{\text {source }} \mathrm{t}_{\text {dispersion }} \mathrm{t}_{\text {efficiency }}
$$

where $\mu_{0}$ is the free space permeability. $t_{\text {source }}=\omega I_{0}(\omega)$ is the sourcedependent term where $\omega=2 \pi f$ is the angular frequency of the generated $\mathrm{THz}$ radiation and $I_{0}(\omega)$ is the Fourier transform of the input optical pump pulse (after accounting for Fresnel reflections at the input interface). This term thus defines the spectral extent of the generated pulse and can be controlled by varying the input pulse duration. As the goal here is to generate $\mathrm{THz}$ in the range 1$5 \mathrm{THz}$, we chose a gaussian pulse with a width $\tau=70 \mathrm{fs}$ (that is FWHM $=165 \mathrm{fs}$ ). This corresponds to asymmetrical gaussian-like pulse centered at $2.27 \mathrm{THz}$ with half maximum intensity points at $0.73 \mathrm{THz}$ and $4.37 \mathrm{THz} . t_{\text {dispersion }}=n\left[n(\omega)+n_{g}\right]^{-1}{ }^{15}$ is the dispersion term directly accounting for the dispersion in the $\mathrm{THz}$ refractive index $n(\omega) . n$ and $n_{g}$ are the refractive index and group index of the optical pulse at the central wavelength $\lambda$ of the pump pulse. The dispersion of the group velocity of the optical pulses is neglected in this work, which is justified by the use of relatively long optical pulses here. $t_{\text {efficiency }}=d_{\text {eff }} L_{\text {eff }}(\omega)$ is the main term that controls the generation efficiency. $d_{\text {eff }}$ is the effective nonlinear coefficient of the nonlinear crystal. $L_{\text {eff }}(\omega)$ is the effective crystal generation length.

Nonlinear properties of Se. The nonlinearity of selenium has been measured at a wavelength of $10.6 \mu \mathrm{m}$, but not at the short IR wavelength used in the present study. Day and coworkers ${ }^{16}$ reported on $d_{\text {eff }}=97 \pm 25 \mathrm{pm} / \mathrm{V}$, citing others showing $d_{\text {eff }}=$ $80 \mathrm{pm} / \mathrm{V}$ and $210 \mathrm{pm} / \mathrm{V}$. Such discrepancy may be attributed to the quality of the crystal or the accuracy of their measurements. However, as Se shows very little refractive index dispersion in the 1-10 $\mu \mathrm{m}$ range, very small dispersion in the electro-optic coefficient $r$ (as confirmed in ref. 17 at $1.5 \mu \mathrm{m}$ in comparison with Teich's ${ }^{18}$ at $10.6 \mu \mathrm{m})$ and $d_{\text {eff }}$ are expected $\left(d_{\text {eff }}=-n^{4} r / 4\right)$. Therefore, as a best estimate, we use $d_{\text {eff }}=97 \mathrm{pm} / \mathrm{V}$ for all the calculations presented here. We mention that triangular Se is birefringent, but only the ordinary beam is considered here.

Effective generation length. The effective crystal length takes into account both phase matching (between the THz phase velocity and the optical pump group velocity) and absorption. When the crystal has weak absorption (such as Se), $L_{\text {eff }}$ is reduced to the commonly used coherence length in transparent $\mathrm{THz}$ generating crystals. Figure 1(a) shows the refractive indices of DAST and Se in the range $0.3-7 \mathrm{THz}$ with main absorption lines at $1.1 \mathrm{THz}$ and 4.2 $\mathrm{THz}$ respectively. In the calculations, we used the frequencydependent refractive indices and absorption coefficients ${ }^{12,14}$. The strong refractive index dispersion around these peaks prevents broadband phase matching. In Fig. 1(b) \& 1(c), $L_{\text {eff }}$ in Se and DAST is shown in the $0.3-4 \mathrm{THz}$ range. As expected from Fig. 1(a), in DAST, $L_{\text {eff }}$ approaches zero at $1.1 \mathrm{THz}$ (as it coincides with the absorption peak) then features a sharp peak (reaching $L_{\text {eff }} \sim$ $820 \mu \mathrm{m})$ at $1.8 \mathrm{THz}$ in a narrow bandwidth. Apart from this, $L_{\mathrm{eff}}$ is on the order of $100-150 \mu \mathrm{m}$ in the spectral region of interest. Selenium shows much better and broader phase matching before the dispersion gets stronger close to the absorption resonance at 4.2 THz. To illustrate this $L_{\text {eff }}$ has minima of $2.1 \mathrm{~mm}, 1.4 \mathrm{~mm}$, $0.5 \mathrm{~mm}$ over spectra extending up to $2.1 \mathrm{THz}, 2.4 \mathrm{THz}, 2.8 \mathrm{THz}$, respectively. While DAST has the highest nonlinear coefficient the superior dielectric properties of Se can over-compensate for that which results in superior spectral density and conversion efficiency.

Design of broadband Se-based source. In order to design a spectrally intense, non-modulated Se-based $\mathrm{THz}$ source, we first
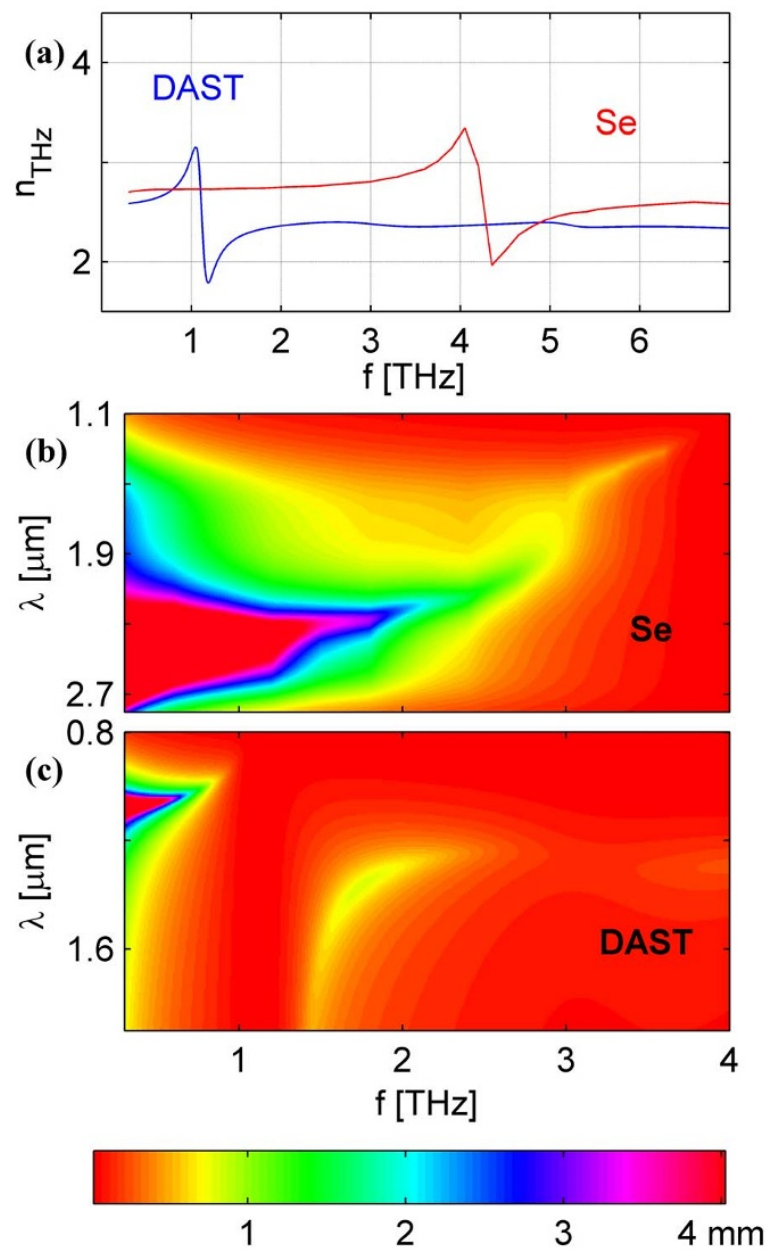

Figure $\mathbf{1} \mid$ (a) The THz refractive index of DAST and Se. The effective crystal generation length from (b) Se and (c) DAST in the 0.3-4 THz range for different pump wavelengths $\lambda$. Selenium offers a much wider phase matching range for both the pump wavelengths and the generated $\mathrm{THz}$ spectra up to crystals with large thickness $\mathrm{d}$ (color code).

seek for the best compromise between the pump wavelength $\lambda$ and the crystal thickness $(d)$ for considerable energy conversion. As shown in Fig. $1(\mathrm{~b})$, we chose $\lambda=\{1.8,2,2.1,2.2,2.3,2.4\} \mu \mathrm{m}$. Figure 2(a) shows the corresponding $\mathrm{THz}$ pulse energies (En) calculated as a function of $d$. For $d<1 \mathrm{~mm}$, these pump wavelengths show comparable conversion efficiencies. At higher thickness, the conversion tends to increase with $\lambda$ until $\lambda=$ $2.3 \mu \mathrm{m}$ before it starts to decrease again for longer wavelengths This behavior reflects the corresponding $L_{\text {eff }}$ shown in Fig. 1(b). Conversely, in DAST, the conversion increases at a much higher rate (driven by the high $d_{\text {eff }}$ ) up to $d \cong 0.65 \mathrm{~mm}$. Then it drops due to the short phase-matched length (Fig. 1(c)). The maximal energies obtained from DAST and Se are comparable. However, the spectral shapes from the two sources are very different. To emphasis the advance of Se in its region of efficiency, we compare its $\mathrm{THz}$ output with $\mathrm{THz}$ emission from DAST, up to $3 \mathrm{THz}$. As expected, while Se shows almost no decrease in its converted energy as function of $\mathrm{d}$, DAST features a drop. At higher $\mathrm{THz}$ frequencies (beyond 3-4 THz), $L_{\text {eff }}$ (and the conversion efficiency) in Se drops significantly.

Figure 2(b) demonstrates further that the corresponding $\mathrm{THz}$ spectrum (at $\lambda=2 \mu \mathrm{m}$ ) is very broadband and barely depends on $d$ in the range of $1.2-2 \mathrm{~mm}$. Using even longer crystals $(>2 \mathrm{~mm})$, however, would lead to a drop in efficiency (see Fig. 2a) due to the 

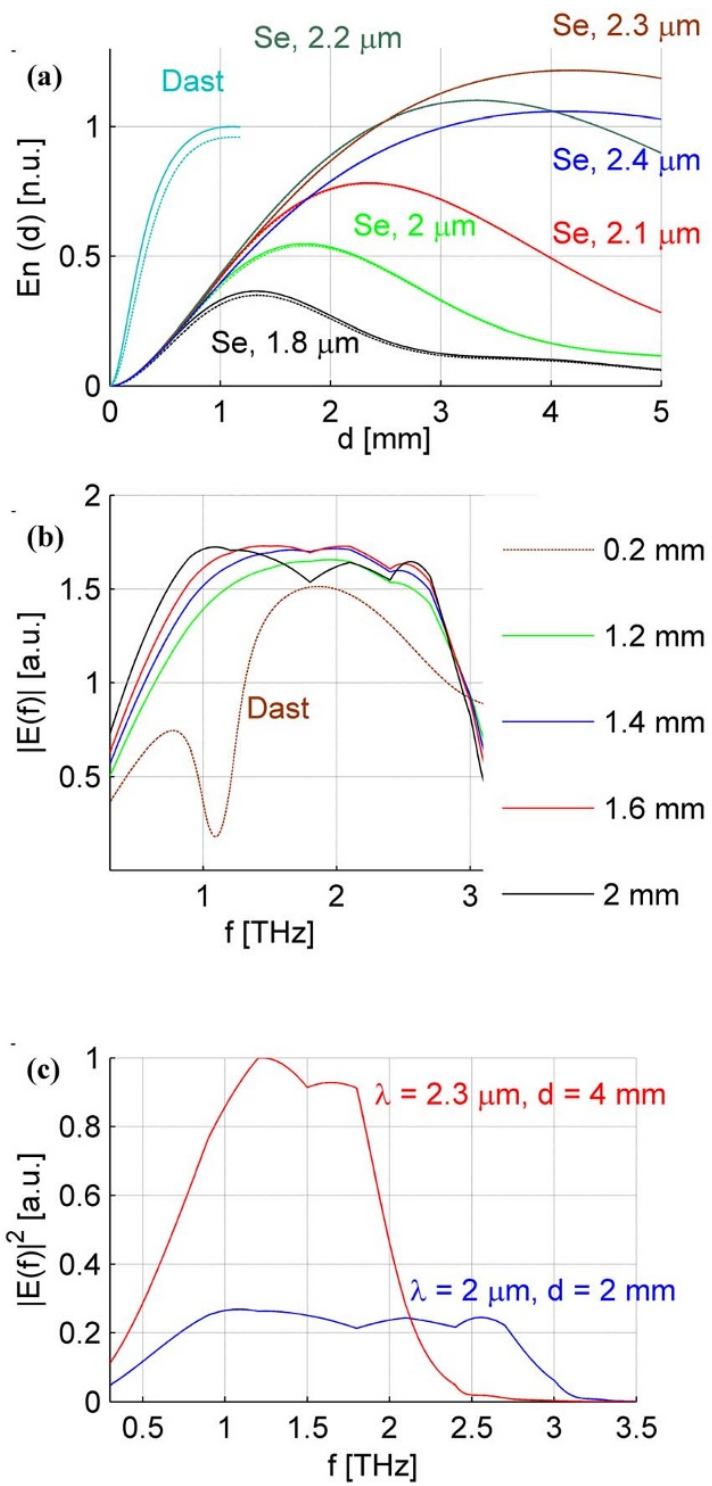

Figure $2 \mid$ (a) The THz pulse energies generated from Se (at various pump wavelengths $\lambda)$ and DAST $(\lambda=1.5 \mu \mathrm{m})$. The solid and dashed lines refer to the total pulse energy and the energy up to $3 \mathrm{THz}$. (b) The THz spectra from DAST (dashed, $\lambda=1.5 \mu \mathrm{m}$ ) and Se (solid, $\lambda=2 \mu \mathrm{m}$ ). The latter is optimized for large bandwidth and smooth spectral output. The legend refers to the crystal thickness $d$. (c) A comparison between the generated spectrum from Se configured for maximum conversion efficiency (red) and broadest spectrum (blue), respectively.

onset of phase mismatch (Fig. 1b). In contrast to the smooth broadband THz spectrum of Se the spectrum of DAST $(d=0.2 \mathrm{~mm})$ shows strong frequency dependent modulations. We conclude that in the range of $0.5-3 \mathrm{THz}$, a Se-based $\mathrm{THz}$ source offers higher spectral densities and flatness than a DAST-based source. We chose $d=0.2 \mathrm{~mm}$ for DAST following the studies presented in ref. 9. Using thinner DAST crystals will hardly improve its flatness as this is mainly controlled by the strong absorption peaks. Longer crystals can increase the spectral density around $2 \mathrm{THz}$ beyond $\mathrm{Se}$, at the expense of the spectral FWHM or flatness.

Next, our simulations suggest that $\lambda=2 \mu \mathrm{m}$ is the most appropriate to produce an ultrabroadband spectral flatness up to $3 \mathrm{THz}$. Although longer $\lambda$ leads to higher energy conversion, this does not necessarily lead to a flat spectrum. If, however, a higher spectral density is requested around a specifically defined central frequency, a different set of pump wavelength and crystal thickness needs to be chosen. For example, Fig. 2a shows that a pump wavelength of $2.3 \mu \mathrm{m}$ of a $4 \mathrm{~mm}$-long Se leads to a much higher efficiency. In Fig. 2c, we compare this case with the case of $2 \mu \mathrm{m}$ pump of $2 \mathrm{~mm}$-long crystal. While the latter provides a flattop spectrum the former offers a Gaussian-like spectrum with a 4 to 5 fold increase in the spectral density, at the expense of the spectral broadness though.

Finally, we look at the corresponding pulse time profile. In some applications such as magnetization dynamics ${ }^{3,6}$, the nonlinear response strongly depends on the time profile of the THz pulse. Figure 3 shows a coherent buildup of the pulse amplitude over $d$ in Se. Except for a cumulative carrier envelope phase, the output electric fields at 0.4 and $2 \mathrm{~mm}$ maintain the original shape while the field strength steadily increases, apart from an additional phase shift due to accumulated material dispersion in the crystal. In contrast, DAST offers an inferior performance as the time profile strongly changes with $d$ and is greatly dispersed. This originates from both the short $L_{\text {eff }}$ and the strong refractive index dispersion (driven by the $1.1 \mathrm{THz}$ resonance absorption, Fig. 1(a)).

Further discussion. In our study, we neglected the effect of two photon absorption (TPA), repetition rate, and pulse duration. There is no report on TPA absorption in Se, but it is expected to be weak in the range of pump photon energy ( $<$ half the energy gap) we used. DAST has a low TPA coefficient of $0.7 \mathrm{~cm} / \mathrm{GW}^{15}$. However, fabricating a DAST crystal with large aperture with good quality is a big technological challenge. Moreover, the merit of high TPA coefficient in DAST is somewhat diminished by the dependence of the damage threshold on the laser repetition rate. In ref. 19, Matsukawa et al show that beyond $40 \mathrm{~Hz}$ (using ns and ps pulses) the damage threshold of DAST deceases with repetition rate. Although this effect is not yet reported in the fs regime, it is practically conceived. In our calculations, we fixed the pulse duration. However, this is not ideal for tunable $\mathrm{THz}$ sources. We
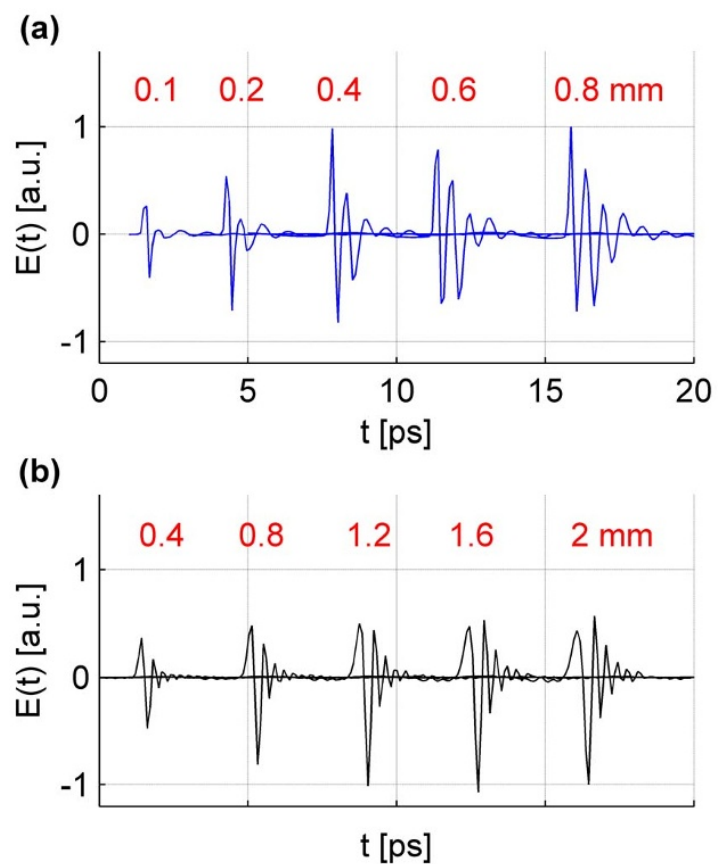

Figure $3 \mid$ The buildup of the temporal profiles along the propagation direction in (a) DAST and (b) Se crystals. While the single-cycle pulse maintains it temporal shape (except for a shift of the absolute phase) throughout a thick crystal (up to $2 \mathrm{~mm}$ ), the THz pulse produced in DAST turns from a single-cycle $(\mathrm{d}=0.1 \mathrm{~mm})$ into a multi-cycle pulse $(\mathrm{d}=0.8 \mathrm{~mm})$ while propagating through the crystal. The pulses are arbitrarily time-delayed for clarity. 
limited our $\mathrm{THz}$ range to a minimum of $0.3 \mathrm{THz}$ due to the lack of information on Se dielectric function. We expect that by using longer pulses, intense low frequency $\mathrm{THz}$ could be produced in Se, which is highly demanded for many applications $s^{6}$. Our speculation is corroborated by the good phase matching trend shown in Fig. 1(b).

In conclusion, we have investigated for the first time triangular Selenium as a broadband Terahertz emitter and demonstrated that $\mathrm{Se}$ is a crystal with a great potential for spectrally intense $\mathrm{THz}$ radiation. Depending on the pump laser wavelength, either broadband flat-top or Gaussian-like spectra were produced in the range 0.53.5 THz. Compared with state of the art $\mathrm{THz}$ emitters the produced spectral density seems higher while similar conversion performance was obtained. We would like to stress that more experimental characterization of both the nonlinear coefficient and dielectric function of Se are needed for a better quantification of the OR process. We also mention that Se is highly anisotropic, which allows even better control of the phase matching than what has been considered here. As Se is transparent and low dispersive in the near and mid infrared, it may offer additional nonlinear applications going beyond the efficient $\mathrm{THz}$ generation presented here.

1. Liu, M. et al. Terahertz-field-induced insulator-to-metal transition in vanadium dioxide metamaterial. Nature 487, 345-348 (2012).

2. Kampfrath, T., Tanaka, K. \& Nelson, K. A. Resonant and nonresonant control over matter and light by intense terahertz transients. Nat. Photonics 7, 680-690 (2013).

3. Vicario, C. et al. Off-resonant magnetization dynamics phase-locked to an intense phase-stable terahertz transient. Nat. Photonics 7, 720-723 (2013).

4. Wu, Z. et al. Intense terahertz pulses from SLAC electron beams using coherent transition radiation. Rev. Sci. Instrum. 84, 022701 (2013).

5. Qi, T., Shin, Y.-H., Yeh, K.-L., Nelson, K. A. \& Rappe, A. M. Collective Coherent Control: Synchronization of Polarization in Ferroelectric $\mathrm{PbTiO}_{3}$ by Shaped $\mathrm{THz}$ Fields. Phys. Rev. Lett. 102, 247603 (2009).

6. Shalaby, M. et al. Terahertz macrospin dynamics in insulating ferrimagnets. Phys. Rev. B 88, 140301(R) (2013).

7. Clerici, M. et al. Wavelength scaling of terahertz generation by gas ionization. Phys. Rev. Lett. 110, 253901 (2013).

8. Hirori, H., Doi, A., Blanchard, F. \& Tanaka, K. Single-cycle terahertz pulses with amplitudes exceeding $1 \mathrm{MV} / \mathrm{cm}$ generated by optical rectification in $\mathrm{LiNbO}_{3}$. Appl. Phys. Lett. 98, 091106 (2011)
9. Hauri, C. P., Ruchert, C., Vicario, C. \& Ardana, F. Strong-field single-cycle THz pulses generated in an organic crystal. Appl. Phys. Lett. 99, 161116 (2011).

10. Vicario, C., Monoszlai, B. \& Hauri, C. P. GV/m Single-Cycle Terahertz Fields from a Laser-Driven Large-Size Partitioned Organic Crystal. Phys. Rev. Lett. 112 213901 (2014).

11. Shalaby, M. \& Hauri, C. P. "Demonstration of a low frequency three-dimensional terahertz bullet with extreme brightness," Nat. Commun. 6, 5976 (2015).

12. Palik, E. D. ed., Handbook of Optical Constants of Solids, vol. 1 (Academic Press, San Diego, CA, 1998)

13. Vodopyanov, K. L. Optical THz-wave generation with periodically-inverted GaAs. Laser Photonics Rev. 2, 11-25 (2008).

14. Cunningham, P. D. \& Hayden, L. M. Optical properties of DAST in the THz range. Opt. Express 18, 23620-23625 (2010).

15. Schneider, A. et al. Generation of terahertz pulses through optical rectification in organic DAST crystals: theory and experiment. J. Opt. Soc. Am. B 23, 1822-1835 (2006).

16. Day, G. W. Linear and nonlinear properties of triangular selenium. Appl. Phys. Lett. 18, 347 (1971)

17. Turner, E., Kaminow, I. \& Kolb, E. Electrooptic effect in trigonal selenium at 1.15 microns. IEEE J. Quant. Electr. 4, 234 (1968).

18. Teich, M. C. \& Kaplan, T. Electrooptic effect in trigonal selenium at $10.6 \mu \mathrm{m}$. IEEE J. Quant. Electr. 2, 702 (1966).

19. Matsukawa, T. et al. Pump-beam-induced optical damage depended on repetition frequency and pulse width in 4-dimethylamino- $N^{\prime}$-methyl-4' -stilbazolium tosylate crystal. Appl. Phys. Lett. 103, 023302 (2013).

\section{Author contributions}

M.S. performed the numerical calculations. C.P.H. supervised the project.

\section{Additional information}

Supplementary information accompanies this paper at http://www.nature.com/ scientificreports

Competing financial interests: The authors declare no competing financial interests.

How to cite this article: Shalaby, M. \& Hauri, C.P. Spectrally intense terahertz source based on triangular Selenium. Sci. Rep. 5, 8059; DOI:10.1038/srep08059 (2015).

This work is licensed under a Creative Commons Attribution-NonCommercialNoDerivs 4.0 International License. The images or other third party material in this article are included in the article's Creative Commons license, unless indicated otherwise in the credit line; if the material is not included under the Creative Commons license, users will need to obtain permission from the license holder in order to reproduce the material. To view a copy of this license, visit http:// creativecommons.org/licenses/by-nc-nd/4.0/ 